\title{
Efeitos da Terapia com Anti-TNF alfa na Pressão Arterial em Pacientes com Hipertensão Resistente: Um Estudo Piloto Randomizado, Duplo-Cego e Controlado por Placebo
}

\author{
Effects of Anti-TNF alpha Therapy on Blood Pressure in Resistant Hypertensive Subjects: A Randomized, \\ Double-Blind, Placebo-Controlled Pilot Study \\ Ana Paula de Faria, ${ }^{1}$ Alessandra M. V. Ritter, ${ }^{1}$ Arthur Santa-Catharina, ${ }^{1}$ Débora P. Souza, ${ }^{1}$ Estephania P. Naseri, ${ }^{1}$ \\ Manoel B. Bertolo, ${ }^{1}$ Mariana Rodrigues Pioli, ${ }^{10}$ Caio C. Carvalho, ${ }^{2}$ Rodrigo Modolo, ${ }^{1}$ Heitor Moreno ${ }^{1}$ \\ Universidade Estadual de Campinas, ${ }^{1}$ Campinas, SP - Brasil \\ Universidade de São Paulo, ${ }^{2}$ Ribeirão Preto, SP - Brasil
}

\section{Resumo}

Fundamento: A citocina fator de necrose tumoral alfa (TNF-a) é elevada na hipertensão resistente (HAR), mas os efeitos dos inibidores de TNF-a nessa população ainda são desconhecidos.

Objetivos: O objetivo deste estudo foi avaliar se uma única dose de infliximabe controlada por placebo reduz a pressão arterial (PA) de forma aguda em pacientes com HAR.

Métodos: Realizamos um estudo cruzado, randomizado, duplo-cego e controlado por placebo em que pacientes com HAR receberam infliximabe ou placebo. O desfecho primário foi a alteração dos níveis de PA média em relação ao basal imediatamente após a infusão, obtida por avaliação hemodinâmica não invasiva contínua, batimento a batimento. Os desfechos secundários incluíram alterações em medidas de PA central, ambulatorial e em consultório, na função endotelial, e nos biomarcadores inflamatórios após 7 dias. O nível de significância aceito foi alfa=0,05.

Resultados: Foram incluídos dez portadores de HAR. O resultado do desfecho primário demonstrou uma redução aguda dos níveis de PA média (média das diferenças \pm desvio padrão $=-6,3 \pm 7,2 \mathrm{mmHg}, p=0,02$ ) em relação ao basal, após o uso de infliximabe, em comparação com o placebo. Os níveis de PA diastólica $(-4,9 \pm 5,5 \mathrm{mmHg}, \mathrm{p}=0,02)$, mas não os níveis de PA sistólica $(-9,4 \pm 19,7 \mathrm{mmHg}, p=0,16)$, reduziram após a infusão de infliximabe. Não foram identificadas diferenças significativas nos demais parâmetros hemodinâmicos, nem nos resultados dos desfechos secundários, com exceção dos níveis de TNF-a, que aumentaram continuamente após o uso de infliximabe. Não foram relatados eventos adversos durante o protocolo.

Conclusões: Uma dose única de infliximabe reduziu os níveis de PA média e diastólica imediatamente após sua infusão, em comparação com placebo em HAR. A terapia com anti-TNF-a foi considerada segura e bem tolerada. Os resultados desse estudo prova de conceito são geradores de hipótese e precisam ser investigados em maior detalhe. (Arq Bras Cardiol. 2021; 116(3):443-451)

Palavras-chave: Hipertensão; Pressão Arterial; Infliximab/uso terapêutico; Ensaio Clínico Controlado Randomizado; Inflamação.

\begin{abstract}
Background: The cytokine tumor necrosis factor-alpha (TNF- $\alpha$ ) is elevated in resistant hypertension (RH), but the effects of a TNF- $\alpha$ inhibitor in this population is unknown.

Objective: The aim of this trial was to evaluate whether a single dose of infliximab controlled by placebo acutely reduces blood pressure (BP) in RH subjects.

Methods: A double-blind, placebo-controlled, crossover trial was conducted, and randomized RH subjects received either infliximab or placebo. The primary endpoint was the change in mean BP levels relative to the baseline immediately after the infusion obtained by continuously beatto-beat non-invasive hemodynamic assessment. Secondary endpoints included changes in office, ambulatory and central BP measurements; endothelial function; and inflammatory biomarkers after 7 days. The level of significance accepted was alpha=0.05.
\end{abstract}

Correspondência: Ana Paula de Faria •

Laboratório de Farmacologia Cardiovascular. Edifício FCM 10, 1a andar. Faculdade de Ciências Médicas - Universidade Estadual de Campinas (FCM-

UNICAMP). CEP: 13083-970. Campinas, SP-Brasil.

E-mail: ana.cabralfaria@gmail.com

Artigo recebido em 15/10/2019, revisado em 04/01/2020, aceito em 09/03/2020

DOI: https://doi.org/10.36660/abc.202190703 
Results: Ten RH subjects were enrolled. The primary endpoint analysis showed an acute decrease in mean BP values (mean of differences \pm standard deviation $=-6.3 \pm 7.2 \mathrm{mmHg}, p=0.02)$ from baseline, after the application of infliximab compared with placebo. Diastolic BP levels $(-4.9 \pm 5.5 \mathrm{mmHg}, p=0.02)$, but not systolic BP levels $(-9.4 \pm 19.7 \mathrm{mmHg}, p=0.16)$, lowered after infliximab infusion. No further significant differences were identified in either the other hemodynamic parameters or in secondary endpoints, except for TNF-a levels, which increased continuously after infliximab infusion. No adverse events were reported during the protocol.

Conclusions: A single-dose of infliximab decreased the mean and diastolic BP levels immediately after its infusion, when compared to the placebo. The anti-TNF-a therapy was found to be safe and well-tolerated. The results of this proof-of-concept are hypothesis-generating and need to be further investigated. (Arq Bras Cardiol. 2021; 116(3):443-451)

Keywords: Hypertension; Blood Pressure; Infliximb/therapeutic use; Randomized Controlled Trial; Inflammation.

Full texts in English - http://www.arquivosonline.com.br

\section{Introdução}

É sabido que a inflamação sistêmica de grau baixo é subjacente à fisiopatologia da hipertensão resistente (HAR) devido à falta de controle de pressão arterial (PA), juntamente com condições coexistentes, tais como obesidade, diabetes tipo 2 (DT2) e síndrome metabólica. Recentemente, nosso grupo de pesquisa explorou o papel das citocinas inflamatórias nessa população de alto risco. Um escore inflamatório alto, incluindo, entre outros, a citocina pró-inflamatória fator de necrose tumoral alfa (TNF- $\alpha$ ), foi proposto a pacientes obesos com HAR em comparação com pacientes obesos com hipertensão controlada. ${ }^{1}$ Além disso, altos níveis de TNF- $\alpha$ foi associado a danos vasculares nesses pacientes com HAR. ${ }^{2}$

Apesar da indicação do uso de inibidores de TNF-a ser bem estabelecida para o tratamento de algumas doenças autoimunes, estudos experimentais e clínicos demonstraram seu uso efetivo para condições relacionadas ao sistema cardiovascular (CV), tais como a prevenção da hipertensão, ${ }^{3}$ e a redução de lesão de órgãos-alvo (LOA). ${ }^{4,5} \mathrm{O}$ infliximabe apresentou benefícios cardiovasculares como um agente neutralizador de TNF- $\alpha$ capaz de reduzir os níveis de PA sistólica (PAS) e o remodelamento cardíaco em ratos espontaneamente hipertensos. ${ }^{6}$

Como o processo inflamatório é parte da HAR e o TNF-a está implicado em disfunções cardiovasculares, o objetivo deste estudo piloto de prova de conceito foi avaliar se uma única dose do inibidor de TNF- $\alpha$, infliximabe, controlada por placebo, reduz os níveis de PA em pacientes com HAR.

\section{Métodos}

\section{Desenho do estudo}

Com o uso de um desenho cruzado de intervenção, randomizado, duplo-cego e controlado por placebo, foram explorados os efeitos agudos do infliximabe, um inibidor de TNF- $\alpha$, e seu comparador, placebo constituído de soro fisiológico, como uma terapia adicional ao tratamento padrão para a população com HAR. Um esquema de randomização em blocos foi criado utilizando-se um código gerado por computador. Os pacientes com HAR foram designados aleatoriamente, na inclusão, a receber ou (1) infusão de placebo seguida de infusão de infliximabe depois de um período de wash-out de 40 dias, ou (2) infliximabe seguido de placebo depois de um período de wash-out de 40 dias. Um farmacêutico alocou, de forma não cega, os voluntários do estudo e manteve consigo os códigos de tratamento até o encerramento do estudo. O enfermeiro que preparou as infusões também o fez de forma não cega. Nenhum dos dois profissionais estava envolvido na coleta, análise ou interpretação dos dados. Um médico fez a inclusão de forma cega dos sujeitos no estudo. Os participantes, o médico avaliador e os pesquisadores que avaliaram os resultados permaneceram de forma cega após a designação das intervenções.

O estudo piloto foi de iniciativa do investigador, desenhado pelos investigadores e não houve apoio de nenhuma entidade comercial. Todos os autores garantiram que os dados e análises estivessem completos e acurados, e certificaram a aderência do estudo ao protocolo.

O estudo foi aprovado pelo Comitê de Ética em Pesquisa (número de aprovação 710.449, CAAE 30811214.9.0000.5404, da Faculdade de Ciências Médicas da Universidade Estadual de Campinas-FCM/UNICAMP, Brasil), e registrado no clinicaltrials. gov (NCT02743390). O estudo foi realizado de acordo com princípios éticos para pesquisas médicas em seres humanos da Associação Médica Mundial (Declaração de Helsinki), e todos os participantes assinaram o termo de consentimento livre e esclarecido antes de serem incluídos no estudo. Também foram seguidas as recomendações do documento Consolidated Standards of Reporting Trials (CONSORT).

\section{População}

Pacientes com diagnóstico confirmado de HAR foram recrutados de uma população pré-selecionada do Ambulatório Especializado em Hipertensão Resistente do Hospital de Clínicas da Universidade Estadual de Campinas (UNICAMP, Campinas, Brasil). A HAR foi definida de acordo com a diretriz da American Heart Association. ${ }^{7}$ Foi realizado um diagnóstico preciso de HAR com um período de acompanhamento clínico de 6 meses para a triagem e a exclusão de causas secundárias de hipertensão [estenose de artéria renal (US-Doppler), feocromocitoma (metanefrinas urinárias e tomografia computadorizada), hiperaldosteronismo primário (relação aldosteronarenina $>20 \mathrm{ng} \mathrm{dl}^{-1}$ por $\mathrm{ng} \mathrm{ml}^{-1} \mathrm{~h}^{-1}$ ), síndrome de Cushing (cortisol e níveis de ACTH), apneia obstrutiva do sono (classificada como "alto risco" no questionário de Berlin)], e pseudorresistência (monitorização ambulatorial da pressão arterial (MAPA) e contagem de comprimidos para excluir a hipertensão do jaleco branco e a não aderência à medicação, respectivamente).

Os critérios de exclusão foram doença cardíaca isquêmica sintomática, função renal comprometida, histórico de acidente vascular, infarto do miocárdio e doenças vasculares 
periféricas, diabetes tipo 1, gravidez, tabagismo, doenças autoimunes, ou contraindicação ao uso de infliximabe. Pacientes não qualificados para o estudo também incluíram os que tinham testes tuberculínicos positivos ou inativos (latentes) ou com radiografia torácica póstero-anterior anormal - avaliada por radiologista e reumatologista especialista em infusão de TNF-a (EP).

\section{Protocolo e avaliações do estudo}

O protocolo foi realizado com os participantes recebendo uma única infusão de infliximabe (Remicade ${ }^{\circledR}, 100$ mg, Janssen-Cilag Farmacêutica Ltda.) na dose de $3 \mathrm{mg} / \mathrm{Kg}$, que foi primeiro reconstituída com $10 \mathrm{~mL}$ de água estéril para injeção e, em seguida, diluída com $250 \mathrm{~mL}$ de cloreto de sódio 0,9\% estéril para injeção, de acordo com as instruções do fabricante; ou recebendo uma única infusão de placebo que consistia $250 \mathrm{~mL}$ de cloreto de sódio 0,9\% estéril para injeção. Os pacientes receberam as infusões intravenosas em um período de 2 horas, com uma vazão de $125 \mathrm{ml} / \mathrm{h}$. Não foi coadministrada nenhuma outra medicação. $\mathrm{O}$ cruzamento para o braço de tratamento seguinte (infusão de placebo ou infliximabe) foi feito depois de um período de wash-out de 40 dias.

As avaliações do estudo incluíam 3 etapas (avaliação no basal, imediatamente após a infusão, e 7 dias após a infusão). Na visita para avaliação do basal (antes das infusões - T0) foram avaliados indicadores antropométricos, as PA de consultório, central (análise de onda de pulso AOP) e níveis de MAPA, e dilatação mediada por fluxo (DMF). Além disso, foram coletadas amostras de sangue para determinar biomarcadores adicionais. Os registros hemodinâmicos não invasivos contínuos batimento a batimento foram avaliados por 15 minutos no basal (T0) e imediatamente após (T1) em ambas as infusões. A PA de consultório e a coleta de sangue também foram examinadas imediatamente após ambas as infusões (T1). Para avaliar a resposta de curto prazo devido à meia-vida longa do infliximabe (aproximadamente 8 dias), ${ }^{8} 7$ dias após as infusões (T2), as PA de consultório, central e MAPA, DMF e a coleta de sangue foram reavaliadas (Figura suplementar).

Depois das avaliações do ensaio, foi respeitado um período de wash-out de 40 dias. Em seguida, o tratamento foi trocado (o que significa que participantes que receberam a primeira infusão de soro fisiológico, depois do período de wash-out de 40 dias, receberam infusão de infliximabe; e os participantes que receberam a primeira infusão de infliximabe, depois do período de wash-out de 40 dias, receberam infusão de soro fisiológico), e as avaliações de ensaio foram repetidas. (Figura suplementar).

Nenhum dos participantes alterou sua medicação anti-hipertensiva durante o período do estudo. Todos os procedimentos iniciaram às 08:00, e os parâmetros foram avaliados após 8 horas de jejum noturno. Depois do protocolo, os pacientes ainda permaneceram sob observação por 1 hora antes de serem liberados. Os participantes foram instruídos a relatar os efeitos colaterais comuns do infliximabe ou qualquer outro evento adverso que eles experimentassem em qualquer momento durante o estudo.

\section{Pressão arterial, função endotelial e avaliações} bioquímicas

A PAS e a PA diastólica (PAD) de consultório foram avaliadas em 3 etapas do estudo - basal, imediatamente após a infusão, e 7 dias após a infusão (infliximabe e placebo) - por um profissional de saúde treinado, de acordo com as diretrizes europeias e brasileiras de hipertensão arterial. Utilizamos um esfigmomanômetro digital validado (HEM-907XL, OMRON Healthcare Inc., Bannockburn, IL, EUA). A PA ambulatorial foi medida em 2 etapas do estudo - basal e 7 dias após a infusão (infliximabe e placebo) e foi realizada utilizando-se um monitor oscilométrico automático (Spacelabs90207, Spacelabs Inc, Redmon, WA). Os pacientes foram instruídos a manter suas atividades normais diárias e a registrar suas atividades de 24 horas em um diário pessoal.

A PAS e PAD, e a pressão de pulso foram avaliadas em 2 etapas do estudo - basal e 7 dias após a infusão (infliximabe e placebo) - e foram determinadas por AOP com o sistema Sphygmocor (Artcor, Sidney, Austrália). ${ }^{9}$ A onda de pulso foi obtida pelo método de tonometria de aplanação da artéria radial. O equipamento também fornece dados adicionais em relação à medição da rigidez arterial pelo índice de incremento (AIx), além do Alx corrigido para a frequência cardíaca de 75 bpm (Alx@75). O Alx é definido pela razão entre as ondas refletidas e de ejeção (onda de pulso que percorre as artérias carótida e femoral).

Os dados da avaliação hemodinâmica não invasiva contínua, batimento a batimento, foram avaliados em 2 etapas do estudo - basal e imediatamente após a infusão (infliximabe e placebo) - e foram obtidos utilizando-se o dispositivo Finometer ${ }^{\circledR}$ (Finapres Medical Systems; Amsterdam, Holanda) e o software Finometer ${ }^{\circledR}$ Beatscope Easy versão 02.10 (Finapres Medical Systems, Amsterdam, Holanda). Um manguito de dimensões apropriadas foi colocado no terceiro ou quarto dedo da mão esquerda e o braço foi deixado em repouso sobre uma mesa, com o paciente sentado. Os níveis de PA sistólica (PAS em mmHg), diastólica (PAD em $\mathrm{mmHg}$ ), e média (PAM em $\mathrm{mm}$ ), débito cardíaco (DC em I/min), e resistência vascular periférica total (RVPT em dyn.s/ $\mathrm{cm}^{-5}$ ) foram registrados durante 15 minutos antes de imediatamente após o protocolo de infusões. Para as análises, foram utilizadas as seções estáveis de registros (os 10 minutos iniciais dos registros foram excluídos das análises). O dispositivo Finometer ${ }^{\circledR}$ utiliza o método de fotopletismografia, e fornece medidas hemodinâmicas confiáveis conforme mostrado anteriormente. ${ }^{10,11}$

A função endotelial foi avaliada em 2 etapas do estudo - basal e 7 dias após a infusão (infliximabe e placebo) - e foi determinada pelo método DMF, de acordo com as diretrizes atuais. ${ }^{12,13}$ Foi utilizado um transdutor vascular linear $(7-12 \mathrm{MHz}$, Toshiba Powervision 6000, Tóquio, Japão) sincronizado com sinal de eletrocardiograma (ECG) no protocolo. Os pacientes, em posição supina, em uma sala silenciosa e com ar-condicionado $\left(22-24{ }^{\circ} \mathrm{C}\right)$, foram submetidos a oclusão da artéria braquial por cinco minutos, utilizando um esfigmomanômetro aneróide. O diâmetro da artéria braquial foi registrado antes e depois da compressão com manguito. A alteração do diâmetro da artéria braquial 
foi expressa como uma porcentagem de alteração relativa ao diâmetro do vaso imediatamente antes da inflação do manguito. O exame da função vascular foi realizado por um único examinador experiente, de forma cega. O coeficiente de variação intraobservador foi de 1,6\%.

Foram coletadas amostras de sangue para avaliação em 3 etapas do estudo - basal, imediatamente após a infusão, e 7 dias após a infusão (infliximabe e placebo) - dos níveis plasmáticos de nitrato/nitrito, biomarcadores inflamatórios, tais como o TNF- $\alpha$, interleucinas-6 (IL-6) e -10 (IL-10), adiponectina, proteína quimiotática de monócitos 1 (MCP1), e os hormônios cortisol e aldosterona. Para as medições de nitrato e nitrito, foi coletado plasma heparinizado e imediatamente misturado com uma solução padrão de nitrito diluída a 5:1 contendo $0.8 \mathrm{M}$ de ferricianeto e $1 \%$ de NP-40. ${ }^{14}$ As amostras foram desproteinizadas com metanol (1:1) e centrifugadas a $14.000 \mathrm{~g}$ por $5 \mathrm{~min}$. Depois disso, foram injetados $300 \mu \mathrm{l}$ de sobrenadante na solução de triiodeto acidificada, que foi purgada com nitrogênio com um analisador de quimiluminescência em fase gasosa de óxido nítrico (ON) (Sievers Model 280i NO Analyzer, Boulder, $\mathrm{CO}, \mathrm{EUA})$. Biomarcadores inflamatórios e hormônios foram medidos em amostras de plasma coletada em EDTA e analisados pelo ensaio de imunoabsorção enzimática (R\&D Systems, Minneapolis, MN, EUA), de acordo com as instruções do fabricante.

\section{Desfechos primário e secundários}

O desfecho primário foi a alteração aguda (de T0 a T1) dos níveis de PA média em relação ao basal imediatamente após a infusão, obtida por avaliação hemodinâmica não invasiva contínua, batimento a batimento.

Os desfechos secundários incluíram alterações em: (1) níveis de PA determinados em consultório em todos os momentos de avaliação do protocolo, pela MAPA e PA central depois de 7 dias; (2) função endotelial após 7 dias; e (3) biomarcadores inflamatórios em todos os momentos de avaliação do protocolo, depois da infusão de infliximabe em comparação com placebo. Todos os resultados secundários foram exploratórios, mas foram considerados relevantes para essa população devido à natureza do ensaio - prova de conceito piloto.

\section{Análises estatísticas}

Foi estimada uma amostra mínima de 10 pacientes com HAR para se detectar uma diferença clínica na PA média de $10 \mathrm{mmHg}$ (desvio padrão de $10 \mathrm{mmHg}$ ) — entre infliximabe e placebo com poder de $80 \%$ e erro tipo alfa de 0,05 .

As variáveis contínuas foram expressas como média e desvio padrão (DP), devido à distribuição normal avaliada pelo teste Kolmogorov-Smirnov. As variáveis categóricas foram apresentadas como frequências e porcentagens. O teste t de Student pareado foi aplicado para comparar os valores de delta (distribuição normal) entre o infliximabe e o placebo nos mesmos pacientes (desenho cruzado). Foi realizado o teste ANOVA de dois fatores para medidas repetidas seguido do teste de comparação múltipla post hoc de Sidak para identificar as diferenças entre os tratamentos (infliximabe $X$ placebo) nos valores delta dos momentos da avaliação (ou seja, T1-T0 - agudo, e T2-T0 - 7 dias).
As análises foram realizadas utilizando-se os softwares SPSS (IBM SPSS Statistics for Mac, Versão 21.0. Armonk, NY: IBM Corp. Lançado em 2012) e GraphPad Prism (versão 7.00 para Windows, GraphPad Software, La Jolla California EUA, www. graphpad.com). O nível de significância aceito foi alfa=0,05.

\section{Resultados}

De março de 2015 a julho de 2017, foram incluídos, no total, 10 pacientes com HAR e todos os pacientes concluíram este ensaio de prova de conceito - Figura 1. As características basais dos pacientes com HAR são apresentadas na Tabela 1. A maioria dos participantes eram do sexo masculino e não brancos. Como o esperado, a maioria era obesa com DT2 e estavam tomando diuréticos e uma grande proporção de $\beta$-bloqueadores, antagonistas dos receptores de angiotensina II, e bloqueadores dos canais de cálcio.

A análise do resultado do desfecho primário demonstrou uma redução aguda dos níveis de PA média em relação ao basal, após o uso de infliximabe, em comparação com o placebo (média das diferenças $\pm \mathrm{DP}$ foi $-6,3 \pm 7,2 \mathrm{mmHg}, \mathrm{p}=0,02$ ). Valores absolutos de delta de PA média em relação ao basal imediatamente após as infusões de placebo e infliximabe nos pacientes estudados são apresentados na Figura 2, e os demais parâmetros hemodinâmicos, na Figura 3. Além da redução dos valores de PA médios, também foi identificada uma redução dos níveis de PAD $(-4,9 \pm 5,5 \mathrm{mmHg}$, $p=0,02)$ em relação ao basal, após o uso de infliximabe, em comparação com o placebo. Não foram identificadas diferenças estatisticamente significativas em PAS, DC e RVPT.

Os resultados dos desfechos secundários são apresentados nas Tabelas suplementares 1 e 2. Não foram identificadas alterações em PA e FC em relação aos tempos delta avaliados, T1-T0 e T2T0, após os tratamentos. Da mesma forma, os níveis plasmáticos não apresentaram nenhuma alteração nem nos parâmetros inflamatórios nem nos hormonais, nos tempos delta avaliados, com exceção do TNF- $\alpha$, que aumentou continuamente após a dose única de infliximabe, em comparação com o placebo (Tabela suplementar 1). Não foram identificadas alterações nos valores delta dos níveis de PA ambulatorial e central 7 dias após as infusões (T2-T0). A função endotelial avaliada por DMF também permaneceu inalterada (Tabela suplementar 2).

Por fim, não foram relatados pelos voluntários eventos adversos durante o protocolo das infusões, nem durante o período do ensaio. Não houve reações alérgicas ao infliximabe, e nenhum paciente abandonou o estudo devido à toxicidade.

\section{Discussão}

O principal achado deste estudo piloto de prova de conceito foi que uma dose única de infliximabe reduziu os níveis de PA média em comparação com placebo, em pacientes com HAR. Secundariamente, o infliximabe também reduziu a PA diastólica. Até onde sabemos, o presente estudo é o primeiro a investigar os efeitos da infusão de um medicamento biológico de anticorpos monoclonais em população portadora de HAR.

Vários estudos clínicos e experimentais demonstraram o papel da citocina pró-inflamatória TNF- $\alpha$ na hipertensão.,15 Uma relação entre o TNF- $\alpha$ e o sistema renina-angiotensina- 


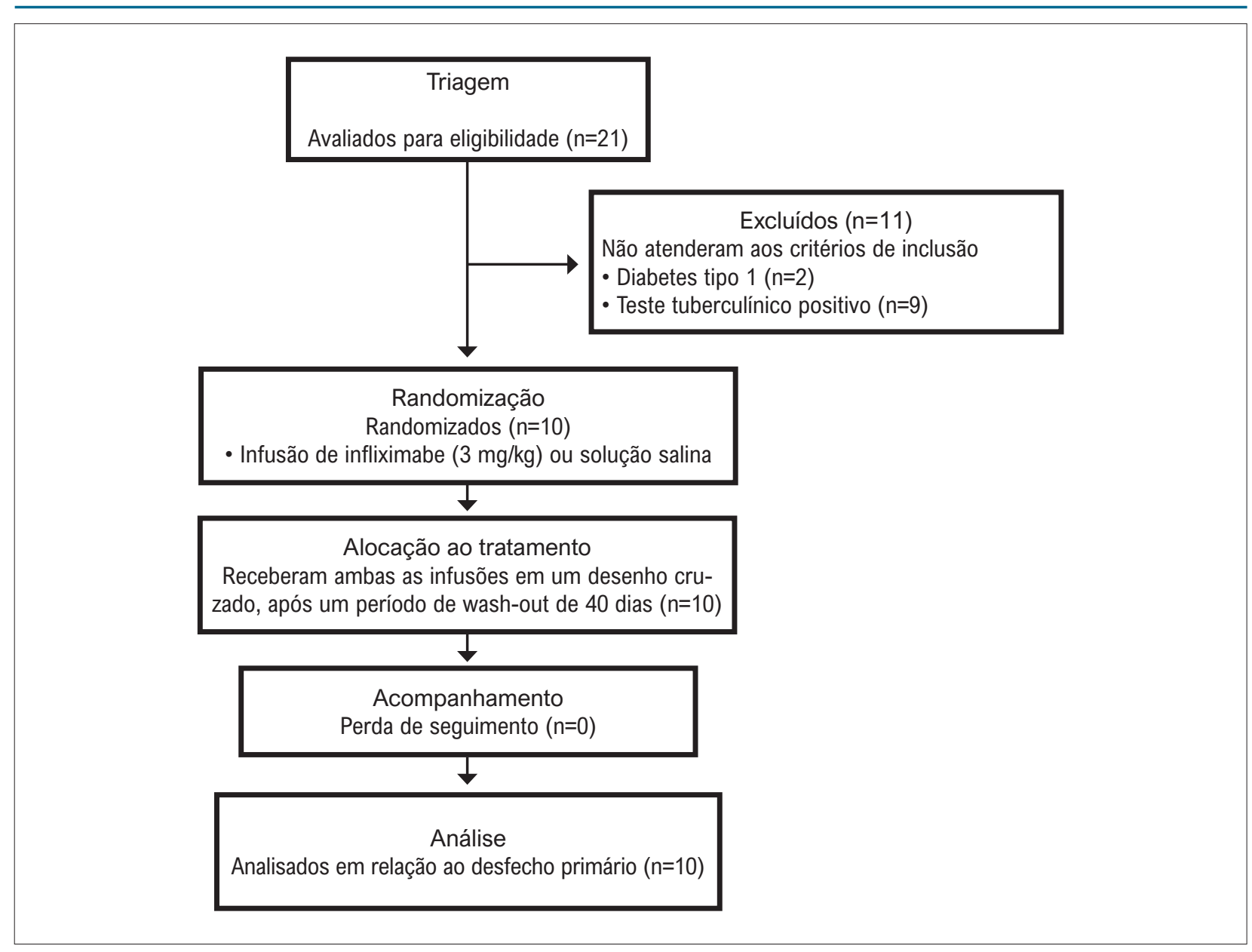

Figura 1 - Fluxograma do estudo.

\section{Tabela 1 - Características da linha de base da população do estudo}

\begin{tabular}{lc} 
& $\begin{array}{c}\text { HAR } \\
\text { (N=10) }\end{array}$ \\
\hline Dados clínicos & $61,8 \pm 8,5$ \\
\hline Idade (anos) & $40 \%(4)$ \\
\hline Sexo (feminino) & $31,9 \pm 5,9$ \\
\hline IMC $\left(\right.$ Kg/m $\left.{ }^{2}\right)$ & $70 \%(7)$ \\
\hline Diabetes tipo 2, $(n)$ & $60 \%(6)$ \\
\hline Não brancos, $(n)$ & $4,4 \pm 0,7$ \\
\hline Medicamentos anti-HA & $100 \%(10)$ \\
\hline Número total & $50 \%(5)$ \\
\hline Diuréticos, $(n)$ & $80 \%(8)$ \\
\hline Espironolactona, $(n)$ & $30 \%(3)$ \\
\hline$\beta$-bloqueadores, $(n)$ & $70 \%(7)$ \\
\hline IECA, $(n)$ & $90 \%(9)$ \\
\hline ARA II, $(n)$ & $12 \%(1)$
\end{tabular}

Os dados foram expressos como média e desvio padrão, ou porcentagem e número absoluto. HAR: hipertensão resistente; IMC: índice de massa corporal; Anti-HA: anti-hipertensivos; IECA: inibidores da enzima conversora de angiotensina; ARA II: antagonistas dos receptores de angiotensina Il; BCC: bloqueadores dos canais de cálcio. 


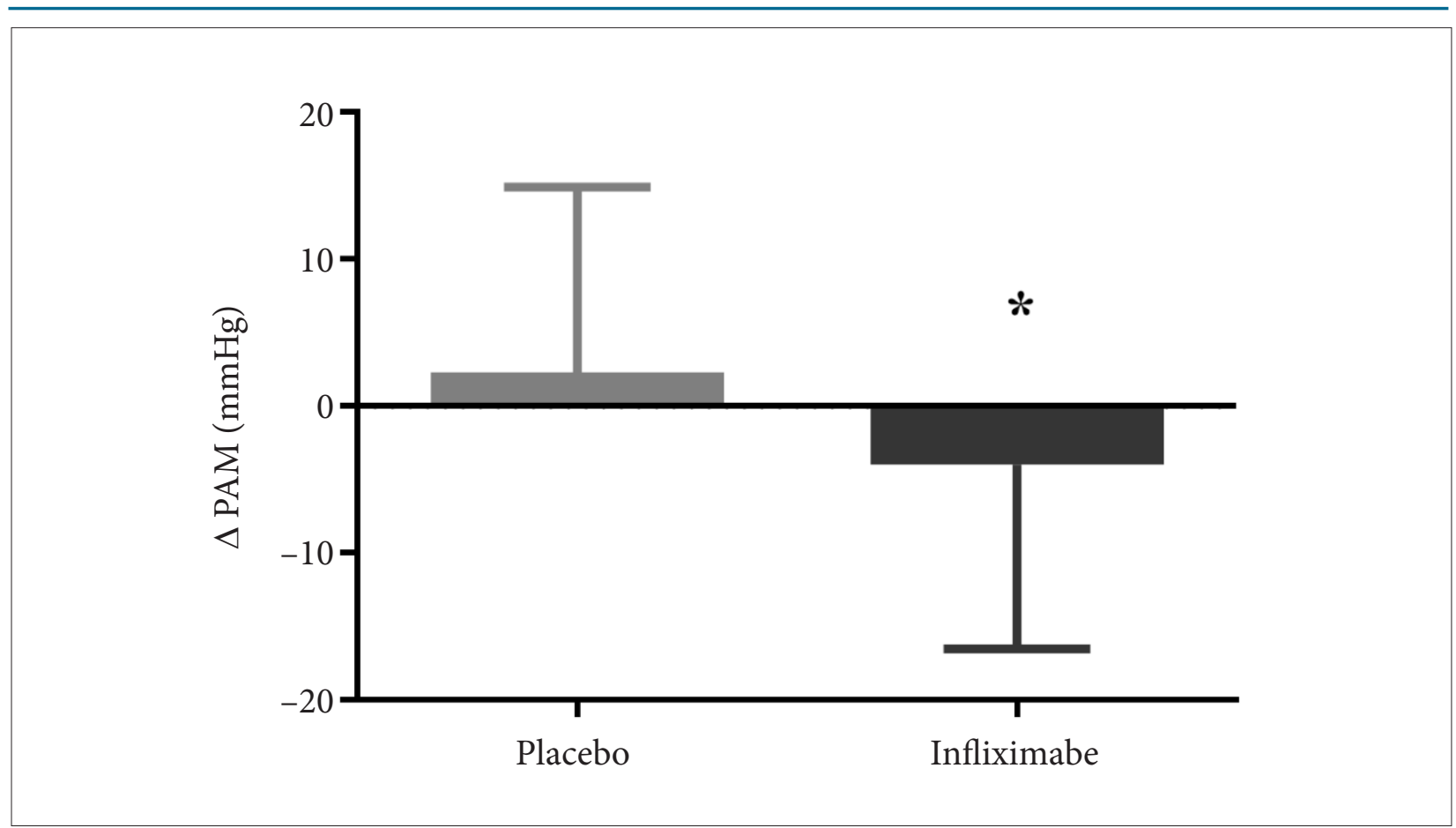

Figura 2 - Delta absoluto da pressão arterial média ( $\triangle P A M, 2,3 \pm 12,6$ versus $-4,0 \pm 12,5 \mathrm{mmHg}$, p=0,02) relativo ao basal imediatamente após (T1-T0) as infusões de placebo e infliximabe, respectivamente, nos pacientes estudados. Os dados foram expressos como média e desvio padrão. 0 teste $t$ de Student pareado foi aplicado para comparar os valores de delta entre o infliximabe e o placebo, * $p<0,05$ versus placebo.

aldosterona (SRAA) foi corroborada pela literatura destacando sua interação na modulação da resposta hipertensiva e da LOA a ela relacionada. ${ }^{3}$ Está bem estabelecido que a HAR com comorbidades existentes, tais como obesidade, DT2 e síndrome metabólica, ${ }^{16,17}$ apresenta hiperativação do sistema nervoso simpático (SNS) e do SRAA. Portanto, presume-se que o TNF-a seria aumentado nessa população de alto risco. Na verdade, este grupo de pesquisa já demonstrou anteriormente que os níveis de TNF- $\alpha$ estão aumentados em HAR em comparação com normotensos, e que essa citocina estava associada ao aumento da rigidez arterial. ${ }^{2}$ Recentemente, demonstrou-se também que um escore inflamatório elevado, combinando várias citocinas circulantes, tais como o TNF- $\alpha$, está relacionado à HAR de maneira dependente da obesidade, em comparação com sujeitos hipertensos controlados. ${ }^{1}$

O uso dos inibidores de TNF-a já foi reconhecido mundialmente para tratamento de doenças autoimunes, especialmente no cenário da reumatologia. ${ }^{18,19} \mathrm{O}$ infliximabe é um medicamento biológico contendo anticorpo monoclonal quimérico humano-murino que neutraliza a atividade do TNF- $\alpha$. Ao realizar uma ligação com alta afinidade às formas solúveis e transmembranares do TNF- $\alpha$, o infliximabe consegue inibir a ligação do TNF- $\alpha$ a seus receptores. ${ }^{20}$ Além dos benefícios clínicos observados após o uso do infliximabe para aliviar os sintomas ou evitar o avanço de doenças autoimunes, sua administração também revelou o potencial para reduzir risco cardiovascular. Por exemplo, resultados de estudos de coorte indicaram a redução na incidência de eventos CV em sujeitos com artrite reumatoide (AR) em uso de terapia anti-TNF- $\boldsymbol{\alpha}^{21,22}$
Os efeitos da inibição de TNF-a para prevenir o aumento dos níveis de PA e lesões a órgãos foram relatados em modelos hipertensivos. ${ }^{6,23}$ Este grupo de pesquisa detectou a redução na PAS e hipertrofia ventricular esquerda em ratos espontaneamente hipertensos após 8 semanas de tratamento com infliximabe (nas doses de 1,5 e 6mg/kg/semana). Esses benefícios cardiovasculares provavelmente foram alcançados devido a um mecanismo dependente de vasodilatação, em que a neutralização de TNF-a foi capaz de induzir a síntese do NO. ${ }^{6}$ No cenário clínico, o infliximabe (inicialmente $3 \mathrm{mg} / \mathrm{Kg}$ a cada 8 semanas durante o período de monitoramento de 1 ano) diminuiu os níveis de PAS e PAD em pacientes com AR recém diagnosticada. ${ }^{24}$ Além disso, depois de 2 semanas de terapia, o infliximabe $(3 \mathrm{mg} / \mathrm{kg})$ reduziu a PA sistólica de 24 horas em pacientes com AR, especialmente durante o período diurno. Este estudo também identificou a redução nos níveis de norepinefrina plasmática e atividade de renina plasmática, sugerindo alterações relacionadas ao infliximabe no SNS e SRAA. ${ }^{25}$ Os achados do presente estudo são, em parte, compatíveis com os de estudos anteriores, pois revelam um efeito modesto de uma dose única da terapia com infliximabe e de $3 \mathrm{mg} / \mathrm{kg}$ na redução dos níveis de PA imediatamente após sua infusão em uma população de risco tão alto como os portadores de HAR. Entretanto, reconhecemos a impossibilidade de atingir a meta de diferença clínica de $10 \mathrm{mmHg}$ projetada neste estudo.

A inibição da via inflamatória pelo infliximabe em nosso estudo pode ter evocado de forma aguda algumas alterações funcionais/bioquímicas, e, consequentemente, acarretado esses níveis de PA reduzidos. Como bem se sabe, o TNF- $\alpha$ é capaz de induzir a disfunção endotelial ${ }^{26}$ (i) estimulando a liberação de micropartículas endoteliais e a produção de espécies reativas 


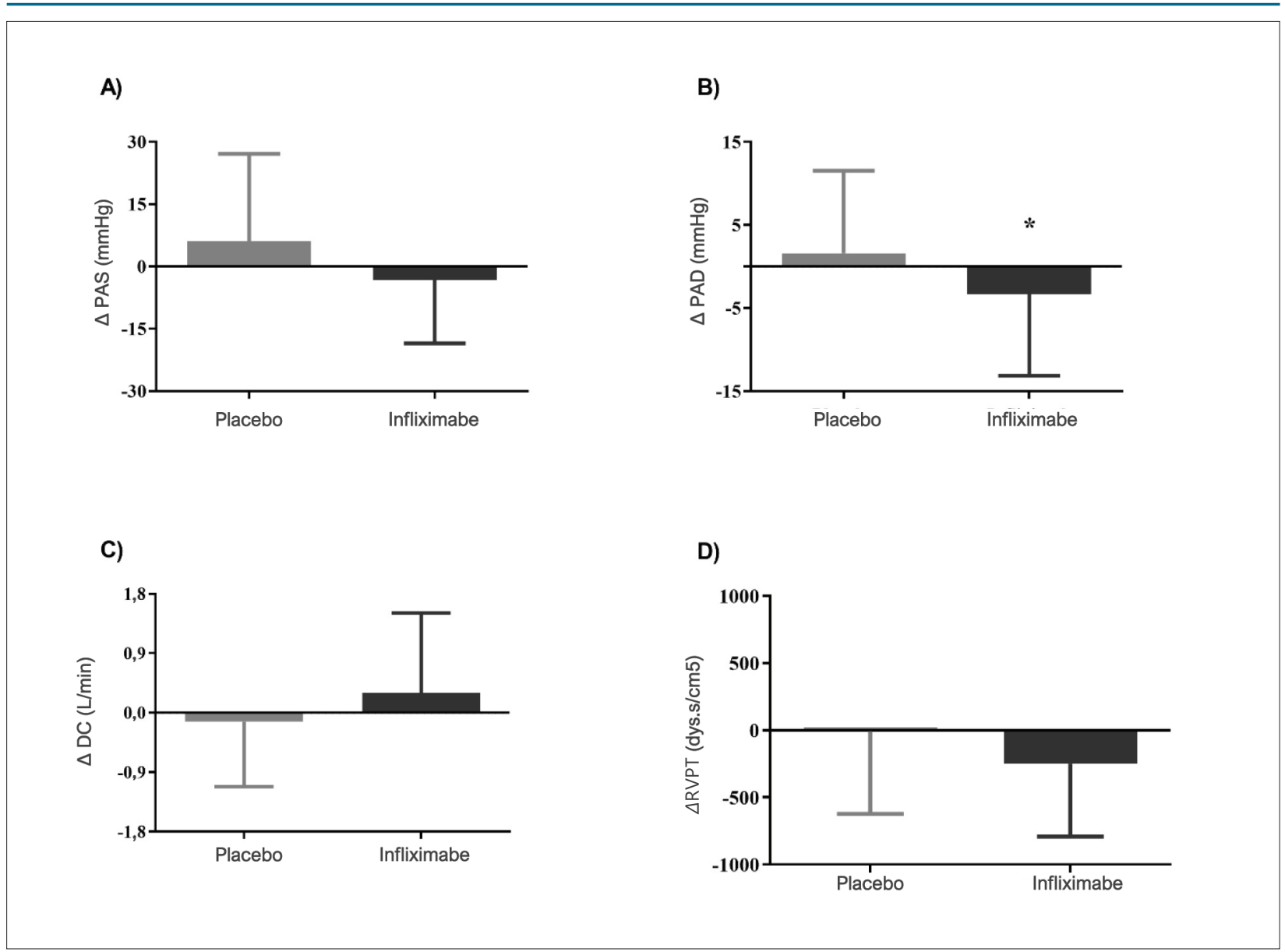

Figura 3 - Deltas absolutos dos parâmetros hemodinâmicos batimento a batimento relativos ao basal imediatamente após (T1-T0) as infusões de placebo e infliximabe, respectivamente, nos pacientes estudados. A. Delta pressão arterial sistólica $(\triangle P A S, 6,1 \pm 21,1$ versus $-3,3 \pm 15,2 \mathrm{mmHg}, p=0,16) ; B$. Delta pressão arterial diastólica ( $\triangle P A D, 1,6 \pm 9,9$ versus $-3,3 \pm 9,8 \mathrm{mmHg}, p=0,02) ; C$. Delta débito cardíaco $(\triangle D C,-0,14 \pm 0,98$ versus 0,30 $\pm 1,21 \mathrm{mmHg}$, $p=0,44) ; D$. Delta resistência vascular periférica total ( $\triangle R V P T,-6,9 \pm 615$ versus $-248 \pm 543 \mathrm{mmHg}$, $p=0,12)$. Os dados foram expressos como média e desvio padrão. 0 teste $t$ de Student pareado foi aplicado para comparar os valores de delta entre 0 infliximabe e o placebo, * $p<0,05$ versus placebo.

do oxigênio, ${ }^{27}$ e (ii) reduzindo a expressão de eNOS constitutiva, ${ }^{28}$ que reduz a biodisponibilidade de NO. Neste estudo, os resultados de DMF não apresentaram diferenças estatísticas 7 dias após a administração aguda de infliximabe, bem como os níveis de metabólitos de NO (nitrato/nitrito) em qualquer um dos dois momentos de avaliação. Embora a inibição de TNF- $\alpha$ tenha sido relatada como possível estratégia para melhorar a função endotelial, ${ }^{29}$ os resultados negativos podem ser explicados, já que foi demonstrado anteriormente que os pacientes com HAR têm vasodilatação gravemente prejudicada associada a maior rigidez vascular ${ }^{30}$ e a níveis mais altos de 8-isoprostano ${ }^{31}$ - um marcador proposto de stress oxidativo in vivo - comparado com hipertensos controlados. Por outro lado, ainda é possível que os níveis de NO imediatamente após o uso de infliximabe estivessem reduzidos em artérias de resistência - que este estudo não conseguiu avaliar - causando as reduções de RVPT e PA, embora esse primeiro parâmetro não tenha sido considerado significativo. Outra hipótese que corrobora nossos achados é a relação entre TNF- $\alpha$ e SRAA, como mencionado acima. Essa citocina pró-inflamatória pode estimular a expressão de receptores de angiotensina tipo $1,{ }^{15}$ e do gene angiotensinogênio no fígado, ${ }^{32}$ este último levando fisiologicamente níveis altos de angiotensina II, e a secreção de aldosterona. Apenas os níveis de aldosterona foram avaliados neste estudo. Embora o resultado tenha permanecido limítrofe e o hormônio possa agir na regulação de longo prazo da PA, foi possível observar uma tendência a uma maior redução de aldosterona nos dois momentos de avaliação após o uso do infliximabe, comparado ao placebo.

É interessante que os níveis de TNF-a tenham aumentado gradualmente nos momentos da avaliação após o uso do infliximabe, em comparação com o placebo. Da mesma forma, alguns estudos detectaram o aumento em seus níveis após a terapia anti-TNF- $\boldsymbol{\alpha},{ }^{29,33}$ embora os mecanismos dessa elevação ainda sejam desconhecidos. Ela possivelmente pode ser explicada pelo prolongamento da meia-vida do TNF- $\alpha$ pelo tratamento - conforme previamente observado nos estudos que exploram outras terapias anti-TNF- $\boldsymbol{\alpha}^{34,35}$ - apesar de ter bloqueado a atividade do TNF- $\boldsymbol{\alpha} .{ }^{34}$ Por outro lado, um tratamento crônico foi associado à redução de níveis do TNF- $\alpha$, atingindo um nível de equilíbrio estável mais baixo, 
o que pode refletir um equilíbrio entre a produção de tecido e a eliminação do TNF- $\alpha .{ }^{36}$ Curiosamente, foi observada uma diminuição aguda dos níveis de PA, que não se manteve após 7 dias de acompanhamento. Como sabemos que neste estudo os níveis de TNF- $\alpha$ aumentaram na semana seguinte à infusão de infliximabe, e que o tratamento crônico tende a diminuir seus níveis, é razoável pressupor que um tratamento crônico poderia ser acompanhado da redução sustentada dos níveis de PA. Entretanto, isso precisa ser provado em um período de acompanhamento mais longo após a infusão.

O presente estudo tem várias limitações inerentes aos estudos piloto de prova de conceito. A limitação mais importante é o tamanho da amostra. É importante mencionar que a população estudada representa um subconjunto muito específico de hipertensos com baixa prevalência global. ${ }^{16}$ Além disso, tivemos uma grande proporção de pacientes com resultados positivos nos testes de tuberculose e, então, foram excluídos do estudo $(n=9)$. Nossos achados negativos em desfechos secundários nos dois momentos de avaliação podem ter acontecido por não ter poder estatístico suficiente (erro tipo II). Uma dose única de $3 \mathrm{mg} /$ $\mathrm{kg}$ pode não ter sido suficiente para causar o efeito clínico esperado imediatamente após a infusão ou qualquer efeito na avaliação de curto prazo (7 dias após a infusão). Embora não tenhamos alcançado a meta de diferença de $10 \mathrm{mmHg}$ na PA média depois do infliximabe em comparação com o placebo [na verdade, a média das diferenças foi de $-6,3$ $(\mathrm{DP}=7,2)$ ], o poder do estudo foi de $70 \%$, e pode, portanto, ser considerado satisfatório, já que este foi um estudo piloto. Um desenho cruzado pode, reconhecidamente, implicar a possível existência de efeitos residuais da medicação. Na tentativa de superar esse problema, e considerando a meiavida longa do infliximabe (a meia-vida de eliminação média é de cerca de 8 dias), ${ }^{8}$ um período de wash-out de 40 dias (5x meias-vidas) seria seguro. Como este estudo utilizou dose única, não é possível garantir que os efeitos da redução dos níveis de PA sejam sustentados durante o período de uso crônico do infliximabe. Por fim, para garantir as validades internas e externas, estudos de larga escala são necessários para estabelecer a segurança clínica e a eficácia de um inibidor de TNF-a no manejo da HAR.

\section{Referências}

1. Faria AP, Ritter AM, Gasparetti CS, Correa NB, Brunelli V, Almeida A, et al. A Proposed Inflammatory Score of Circulating Cytokines/Adipokines Associated with Resistant Hypertension, but Dependent on Obesity Parameters. Arq Bras Cardiol. 2019; 112(4):383-9.

2. Barbaro NR, Fontana V, Modolo R, De Faria AP, Sabbatini AR, Fonseca FH et al. Increased arterial stiffness in resistant hypertension is associated with inflammatory biomarkers. Blood Press 2015; 24(1):7-13.

3. Guzik TJ, Hoch NE, Brown KA, McCann LA, Rahman A, Dikalov S, et al. Role of the T cell in the genesis of angiotensin II induced hypertension and vascular dysfunction. J Exp Med. 2007; 204(10):2449-60.

4. Maki-Petaja KM, Hall FC, Booth AD, Wallace SM, Yasmin, Bearcroft PW, et al. Rheumatoid arthritis is associated with increased aortic pulse-wave velocity, which is reduced by anti-tumor necrosis factor-alpha therapy. Circulation 2006; 114(11):1185-92.

\section{Conclusões}

Este estudo piloto prova de conceito demonstrou que uma dose única de infliximabe reduziu os níveis de PA média e diastólica imediatamente após sua infusão, em comparação com placebo. A terapia com anti-TNF- $\alpha$ foi considerada segura e bem tolerada. Ela acrescenta uma perspectiva clínica às terapias direcionadas ao processo inflamatório para tratar da hipertensão de difícil controle. Devido ao tamanho pequeno da amostra e ao poder abaixo do pré-especificado, os achados devem ser interpretados como exploratórios e geradores de hipótese.

\section{Agradecimentos}

Os autores agradecem ao Centro de Medicamentos de Alta Complexidade (CEDMAC) - Hospital das Clínicas/Universidade Estadual de Campinas (UNICAMP) pelo apoio técnico e por fornecer a medicação.

\section{Contribuição dos autores}

Concepção e desenho da pesquisa: Faria AP, Modolo R, Moreno $\mathrm{H}$; Obtenção de dados e Revisão crítica do manuscrito quanto ao conteúdo intelectual importante: Faria AP, Ritter AMV, Santa-Catharina A, Souza DP, Naseri EP, Bertolo MB, Pioli MR, Carvalho CC, Modolo R, Moreno H; Análise e interpretação dos dados: Faria AP, Ritter AMV, Santa-Catharina A, Modolo R; Análise estatística e Redação do manuscrito: Faria AP; Obtenção de financiamento: Faria AP, Bertolo MB, Moreno $\mathrm{H}$.

\section{Potencial conflito de interesses}

Declaro não haver conflito de interesses pertinentes.

\section{Fontes de financiamento}

O presente estudo foi financiado pela FAPESP (2015/17151-7) e parcialmente financiado pelo CNPq e CAPES.

\section{Vinculação acadêmica}

Não há vinculação deste estudo a programas de pósgraduação.

5. Maki-Petaja KM, Elkhawad M, Cheriyan J, Joshi FR, Ostor AJ, Hall FC, et al. Antitumor necrosis factor-alpha therapy reduces aortic inflammation and stiffness in patients with rheumatoid arthritis. Circulation. 2012; 126(21):2473-80.

6. Filho AG, Kinote A, Pereira DJ, Renno A, dos Santos RC, Ferreira-Melo SE, et al. Infliximab prevents increased systolic blood pressure and upregulates the AKT/eNOS pathway in the aorta of spontaneously hypertensive rats. Eur J Pharmacol. 2013;700(1-3): 201-9.

7. Calhoun DA, Jones D, Textor S, Goff DC, Murphy TP, Toto RD, et al. Resistant hypertension: diagnosis, evaluation, and treatment. A scientific statement from the American HeartAssociation Professional Education Committee of the Council for High Blood Pressure Research. Hypertension. 2008; 51(6):1403-19.

8. Kavanaugh A, St Clair EW, McCune WJ, Braakman T, Lipsky P. Chimeric antitumor necrosis factor-alpha monoclonal antibody treatment of patients with rheumatoid arthritis receiving methotrexate therapy. J Rheumatol. 2000; 27(4):841-50. 
9. LaurentS, CockcroftJ, Van Bortel L, Boutouyrie P, Giannattasio C, Hayoz D, etal. European Network for Non-invasive Investigation of Large A, Expert consensus document on arterial stiffness: methodological issues and clinical applications. Eur Heart J.2006; 27(21):2588-605.

10. Imholz BP, van Montfrans GA, Settels JJ, van der Hoeven GM, Karemaker JM, Wieling W. Continuous non-invasive blood pressure monitoring: reliability of Finapres device during the Valsalva manoeuvre. Cardiovasc Res. 1988; 22(6):390-7.

11. Imholz BP, Langewouters GJ, van Montfrans GA, Parati G, van Goudoever J, Wesseling KH, etal. Feasibility of ambulatory, continuous 24-hour finger arterial pressure recording. Hypertension 1993; 21(1):65-73.

12. Corretti MC, Anderson TJ, Benjamin EJ, Celermajer D, Charbonneau F, Creager MA, et al. Guidelines for the ultrasound assessment of endothelial-dependent flow-mediated vasodilation of the brachial artery: a report of the International Brachial Artery Reactivity Task Force. J Am Coll Cardiol 2002; 39(2):257-65.

13. Thijssen DH, BlackMA, Pyke KE, Padilla J, Atkinson G, Harris RA, etal. Widlansky ME, Tschakovsky ME, Green DJ, Assessment of flow-mediated dilation in humans: a methodological and physiological guideline. Am J Physiol Heart Circ Physiol.. 2011; 300(1):H2-12.

14. Dejam A, Hunter CJ, Pelletier MM, Hsu LL, Machado RF, Shiva S, et al. Erythrocytes are the major intravascular storage sites of nitrite in human blood. Blood. 2005;106(2):734-9.

15. Sriramula S, Haque M, Majid DS, Francis J. Involvement of tumor necrosis factor-alpha in angiotensin II-mediated effects on salt appetite, hypertension, and cardiac hypertrophy. Hypertension 2008; 51(5):1345-51.

16. Carey RM, Calhoun DA, Bakris GL, Brook RD, Daugherty SL, DennisonHimmelfarb CR, et al. American Heart Association Professional/Public E, Publications Committee of the Council on H, Council on C, StrokeN, Council on Clinical C, Council on G, Precision M, Council on Peripheral Vascular D, Council on Quality of C, Outcomes R, Stroke C, Resistant Hypertension: Detection, Evaluation, and Management: A Scientific Statement From the American Heart Association. Hypertension ..2018; 72: e53-e90.

17. Catharina AS, Modolo R, Ritter AM, Sabbatini AR, Lopes HF, Moreno Junior $\mathrm{H}$, et al. Metabolic Syndrome-Related Features in Controlled and Resistant Hypertensive Subjects. Arq Bras Cardiol 2018; 110(6):514-21.

18. Lipsky PE, van der Heijde DM, St Clair EW, Furst DE, Breedveld FC, Kalden JR, etal. Anti-Tumor Necrosis Factor Trial in Rheumatoid Arthritis with Concomitant Therapy Study G, Infliximab and methotrexate in the treatment of rheumatoid arthritis. Anti-Tumor Necrosis Factor Trial in Rheumatoid Arthritis with Concomitant Therapy Study Group. N Engl J Med. 2000; 343(22):1594-602.

19. Smolen JS, Van Der Heijde DM, St Clair EW, Emery P, Bathon JM, Keystone E, et al. Active-Controlled Study of Patients Receiving Infliximab for the Treatment of Rheumatoid Arthritis of Early Onset Study G, Predictors of joint damage in patients with early rheumatoid arthritis treated with high-dose methotrexate with or without concomitant infliximab: resultsfrom the ASPIRE trial. Arthritis Rheum. 2006; 54(3):702-10.

20. Scallon BJ, Moore MA, Trinh H, KnightDM, GhrayebJ. Chimeric anti-TNF-alpha monoclonal antibody CA2 binds recombinant transmembrane TNF-alpha and activates immune effector functions. Cytokine. 1995; 7(3):251-9.

21. Jacobsson LT, Turesson C, Gulfe A, Kapetanovic MC, Petersson IF, Saxne T, et al. Treatment with tumor necrosis factor blockers is associated with a lower incidence of first cardiovascular events in patients with rheumatoid arthritis. J Rheumatol. 2005: 32(7):1213-8
22. Greenberg JD, Kremer JM, Curtis JR, Hochberg MC, Reed G, Tsao P, et al. Investigators $\mathrm{C}$, Tumour necrosis factor antagonist use and associated risk reduction of cardiovascular events among patients with rheumatoid arthritis. Ann Rheum Dis 2011; 70(4):576-82.

23. Abdelrahman AM, Al Suleimani YM, Ashique M, Manoj P, Ali BH. Effect of infliximab and tocilizumab on fructose-induced hyperinsulinemia and hypertension in rats. Biomed Pharmacother. 2018; 105: 182-6.

24. Klarenbeek NB, van der Kooij SM, Huizinga TJ, Goekoop-Ruiterman YP, Hulsmans HM, van Krugten MV, et al. Allaart CF, Blood pressure changes in patients with recent-onset rheumatoid arthritis treated with four different treatment strategies: a posthoc analysis from the BeSttrial. Ann Rheum Dis 2010; 69(7):1342-5.

25. Yoshida S, Takeuchi T, Kotani T, Yamamoto N, Hata K, Nagai K, et al. Infliximab, a TNF-alpha inhibitor, reduces 24-h ambulatory blood pressure in rheumatoid arthritis patients. J Hum Hypertens. 2014; 28(3):165-9.

26. Bhagat K, Vallance P. Inflammatory cytokines impair endothelium-dependent dilatation in human veins in vivo. Circulation, 1997; 96(9):3042-7.

27. Lee SK, Yang SH, Kwon I, Lee $\mathrm{OH}$, Heo JH. Role of tumour necrosis factor receptor-1 and nuclear factor-kappaB in production of TNF-alpha-induced pro-inflammatory microparticles in endothelial cells. Thromb Haemost 2014; 112(3):580-8.

28. Yoshizumi M, Perrella MA, Burnett Jr JC, Lee ME. Tumor necrosis factor downregulates an endothelial nitric oxide synthase mRNA by shortening its halflife. Circ Res. 1993; 73(1):205-9.

29. Moreau KL, Deane KD, Meditz AL, Kohrt WM. Tumor necrosis factor-alpha inhibition improves endothelial function and decreases arterial stiffness in estrogen-deficient postmenopausal women. Atherosclerosis. 2013; 230(2):390-6.

30. Figueiredo VN, Yugar-Toledo JC, Martins LC, Martins LB, de Faria AP, de Haro Moraes $\mathrm{C}$, et al. Vascular stiffness and endothelial dysfunction: Correlations at different levels of blood pressure. Blood Press 2012; 21(1):31-8.

31. de Faria AP, Fontana V, Modolo R, Barbaro NR, Sabbatini AR, Pansani IF, et al, Plasma 8-isoprostane levels are associated with endothelial dysfunction in resistant hypertension. Clin Chim Acta 2014; 433: 179-83.

32. Ferreri NR, Zhao Y, Takizawa H, McGiff JC. Tumor necrosis factor-alphaangiotensin interactions and regulation of blood pressure. J Hypertens. 1997; 15(12 Pt 1): 1481-4

33. Tsimberidou AM, Waddelow T, Kantarjian HM, Albitar M, Giles FJ. Pilot study of recombinant human soluble tumor necrosis factor (TNF) receptor (p75) fusion protein (TNFR:Fc; Enbrel) in patients with refractory multiple myeloma: increase in plasma TNF alpha levels during treatment. Leuk Res. 2003; 27(5):375-80.

34. Eason JD, Pascual M, Wee S, Farrell M, Phelan J, Boskovic S, et al. Evaluation of recombinant human soluble dimeric tumor necrosis factor receptor for prevention of OKT3-associated acute clinical syndrome. Transplantation. 1996; 61(2):224-8

35. Mohler KM, Torrance DS, Smith CA, Goodwin RG, Stremler KE, FungVP, Madani $\mathrm{H}$, Widmer MB, Soluble tumor necrosis factor (TNF) receptors are effective therapeutic agents in lethal endotoxemia and function simultaneously as both TNF carriers and TNF antagonists. J Immunol 1993; 151(3):1548-61.

36. Madhusudan S, Foster M, Muthuramalingam SR, BraybrookeJP, Wilner S, Kaur $\mathrm{K}$, et al. A phase II study of etanercept (Enbrel), a tumor necrosis factor alpha inhibitor in patients with metastatic breast cancer. Clin Cancer Res.. 2004; 10(19):6528-34.

* Material suplementar

Para informação adicional, por favor, clique aqui. 